\title{
Sequence variation in human succinate dehydrogenase genes: evidence for long-term balancing selection on SDHA Bora E Baysal ${ }^{* 1,2}$, Elizabeth C Lawrence ${ }^{2}$ and Robert E Ferrell ${ }^{2}$
}

Address: ${ }^{1}$ Department of Obstetrics, Gynecology and Reproductive Sciences, University of Pittsburgh School of Medicine, Pittsburgh, PA 15213, USA and ${ }^{2}$ Department of Human Genetics, Graduate School of Public Health, University of Pittsburgh, Pittsburgh, PA 15213, USA

Email: Bora E Baysal* - baysalb@mwri.magee.edu; Elizabeth C Lawrence - elawrenc+@pitt.edu; Robert E Ferrell - rferrell@hgen.pitt.edu

* Corresponding author

Published: 2I March 2007

BMC Biology 2007, 5:12 doi:10.1/86/1741-7007-5-12

Received: 9 September 2006

Accepted: 21 March 2007

This article is available from: http://www.biomedcentral.com/I741-7007/5/12

(C) 2007 Baysal et al; licensee BioMed Central Ltd.

This is an Open Access article distributed under the terms of the Creative Commons Attribution License (http://creativecommons.org/licenses/by/2.0), which permits unrestricted use, distribution, and reproduction in any medium, provided the original work is properly cited.

\begin{abstract}
Background: Balancing selection operating for long evolutionary periods at a locus is characterized by the maintenance of distinct alleles because of a heterozygote or rare-allele advantage. The loci under balancing selection are distinguished by their unusually high polymorphism levels. In this report, we provide statistical and comparative genetic evidence suggesting that the SDHA gene is under long-term balancing selection. SDHA encodes the major catalytical subunit (flavoprotein, $\mathrm{FP}$ ) of the succinate dehydrogenase enzyme complex (SDH; mitochondrial complex II). The inhibition of $\mathrm{Fp}$ by homozygous SDHA mutations or by 3nitropropionic acid poisoning causes central nervous system pathologies. In contrast, heterozygous mutations in SDHB, SDHC, and SDHD, the other SDH subunit genes, cause hereditary paraganglioma (PGL) tumors, which show constitutive activation of pathways induced by oxygen deprivation (hypoxia).
\end{abstract}

Results: We sequenced the four SDH subunit genes (10.8 kb) in 24 African American and 24 European American samples. We also sequenced the SDHA gene $(2.8 \mathrm{~kb})$ in 18 chimpanzees. Increased nucleotide diversity distinguished the human SDHA gene from its chimpanzee ortholog and from the PGL genes. Sequence analysis uncovered two common SDHA missense variants and refuted the previous suggestions that these variants originate from different genetic loci. Two highly dissimilar SDHA haplotype clusters were present in intermediate frequencies in both racial groups. The SDHA variation pattern showed statistically significant deviations from neutrality by the Tajima, $\mathrm{Fu}$ and $\mathrm{Li}$, Hudson-Kreitman-Aguadé, and Depaulis haplotype number tests. Empirically, the elevated values of the nucleotide diversity $(\% \pi=0.231)$ and the Tajima statistics $(D=1.954)$ in the SDHA gene were comparable with the most outstanding cases for balancing selection in the African American population.

Conclusion: The SDHA gene has a strong signature of balancing selection. The SDHA variants that have increased in frequency during human evolution might, by influencing the regulation of cellular oxygen homeostasis, confer protection against certain environmental toxins or pathogens that are prevalent in Africa. 


\section{Background}

Succinate dehydrogenase (SDH; mitochondrial complex II) is an essential enzyme complex that has dual roles in the Krebs cycle and the electron transport chain (ETC) in mitochondria [1]. SDH is composed of four subunits encoded by the nuclear genes $S D H A, S D H B, S D H C$, and $S D H D$. SDHA at chromosome band 5p15 and SDHB at chromosome band 1p35 encode the two catalytical hydrophilic subunits flavoprotein (Fp; $70 \mathrm{kDa}$ ) and ironsulfur (Ip; $35 \mathrm{kDa}$ ), respectively. SDHC at chromosome band $1 \mathrm{q} 23$ and SDHD at chromosome 11q23 encode the two membrane-spanning hydrophobic subunits, cybL (15 $\mathrm{kDa})$ and cybS $(12 \mathrm{kDa})$, respectively. The $S D H A, S D H B$, $S D H C$, and $S D H D$ gene products are encoded by $15,8,6$, and 4 exons, which span genomic distances of $\sim 38 \mathrm{~kb}, 35$ $\mathrm{kb}, 50 \mathrm{~kb}$. and $10 \mathrm{~kb}$, respectively $[2,3]$.

The identification of the SDHD subunit gene as the hereditary paraganglioma type 1 locus (PGL1) has uncovered unexpected links between SDH and tumor susceptibility, and highlighted the role of mitochondria in cancer [4]. Since then, mutations in $S D H B, S D H C$, and $S D H D$ subunit genes (PGL genes) have been established as an important cause of sporadic and familial paragangliomas [5-10]. The paraganglia specificity of PGL tumors [4] and data from global gene-expression analysis [11], cell biology [12], animal-model studies [13], and gene-environment interaction and population genetics [14] support the hypothesis that constitutive hypoxic stimulation underlies the pathogenesis of PGL.

The role of SDH in disease pathogenesis has been implicated independently through a series of studies on a widely distributed plant and fungal neurotoxin, 3-nitropropionic acid (3-NPA)[15]. Acute food poisoning with 3-NPA, which can lead to central nervous system defects with lifelong disability and to mortality in $\sim 10 \%$ of the cases, have been associated with consumption of moldy sugarcanes in China [16]. The neurodegeneration induced by 3-NPA poisoning often involves the basal ganglia, hippocampus, spinal tracts, and peripheral nerves, and the symptoms mimic those of Huntington's disease [17]. 3NPA irreversibly inhibits SDH, owing to the similarity of the chemical structures of 3-NPA to succinate [18]. It has been suggested that 3-NPA may form a covalent adduct with an arginine residue at amino acid position 345 in the active site of the Fp subunit [19].

Surprisingly, mutations in the major catalytical subunit SDHA have yet to be associated with PGL. Although homozygous mutations in SDHA have been found in Leigh syndrome [20], a severe neurodegenerative disorder of childhood, and with neuromusculopathies, no genetic link between SDHA and paraganglioma susceptibility has ever been established. Current biochemical knowledge on
SDH provides very few clues for the phenotypic dichotomy arising from the germline subunit gene mutations. SDHA and SDHB subunits encode the two physicallyinteracting catalytical subunits, so it is surprising that their mutations would have such different phenotypic consequences [21]. Recently, after identifying cDNA sequences encoding a missense Fp variant containing the Y629F and V657I polymorphisms, Tomitsuka et al $[22,23]$ proposed that distinct genetic loci encode two Fp variants, namely type I and type II. They reached this conclusion after observing tissue-specific and cell line-specific differential expression of the CDNA variants and PCR amplification from genomic DNA of processed SDHA gene fragments that lacked introns (i.e, a functional SDHA retrogene). However, the genomic location of the retrogene that was proposed to encode the second SDHA gene could not be determined. A retrogene for SDHA is not present in the human genome, according to the March 2006 assembly in The UCSC database. [24] Finally, Briere et al [25] showed the presence of the missense SDHA variants in several different cell types and assumed that these variants originate from two different genes, although they provided no experimental or bioinformatic evidence for the genomic presence of a second SDHA locus. Briere et al [25] suggested that the presence of two SDHA genes in paraganglia prevents tumorigenesis. If Fp were encoded by two different loci, this would indeed have provided a simple explanation for why SDHA mutations would not be associated with PGL susceptibility.

An alternative approach to gain insights into gene function involves analysis of sequence variation in the population. To date, no study has systematically addressed the variation patterns in the SDH subunit genes in normal subjects from different racial or ethnic groups. To gain further insights into the multiple roles of SDH in disease predisposition and to help to integrate the seemingly disparate phenotypic consequences of SDH subunit defects, we examined sequence variation in the complete coding and partial flanking intronic sequences of the four SDH subunit genes in 24 samples from an African American population and 24 samples from a white population. These analyses uncovered an unexpected degree of nucleotide diversity in the SDHA gene.

\section{Results}

\section{Sequence variants in the SDH subunit genes}

Using PCR, each coding exon and the flanking introns of the four SDH subunit genes in 24 European American and 24 African American samples were amplified, and were then sequenced. In total, 3828 coding and 7013 non-coding nucleotides were sequenced for each sample, and 52 polymorphisms were detected (Table 1). The heterozygous frequencies of all variants were consistent with Hardy-Weinberg expectations $(p>0.01)$ in both sets of 
samples. Except for two non-coding indels in SDHA and one in $S D H C$, all variants were single nucleotide polymorphisms (SNPs) involving base replacements. A full list of the identified sequence variants is provided in Additional File 1 and has also been submitted to the SDH mutation database [26]. SDHA variant density was 2.6-fold and 2.3fold higher in the coding and non-coding regions, respectively, than the average of 106 genes [27]. The minor allele frequencies of all variants are shown in Figure 1.

\section{Nucleotide diversity in SDH subunit genes}

We calculated the nucleotide diversity in SDH subunit genes using the population genetic parameters $\pi$ and $\theta_{\mathrm{s}}$ (Table 2). As expected, all diversity indices were higher in the African American samples. The nucleotide diversity (\%) in the total sample set was low at the PGL genes: $\operatorname{SDHB}(\pi=0.008), \operatorname{SDHC}(\pi=0.065)$, and SDHD $(\pi=$ $0.044)$. In contrast, the nucleotide diversity of the SDHA gene $(\pi=0.199 \%)$ was 5.1 -fold higher than the average of the PGL genes and 3.4-fold higher than the average $(\pi=$ $0.058 \%$ ) of 292 autosomal genes [28]. The $\theta_{\mathrm{s}}$ and $\pi$ estimates of nucleotide diversities were similar for the membrane-spanning subunits $S D H C$ and $S D H D$, but differed substantially for the two catalytic subunits. Whereas the $\pi$ estimate was $\sim 1.6$-fold higher than $\theta_{\text {s }}$ for the SDHA gene, consistent with the enrichment of alleles with intermediate frequencies, the $\theta_{\mathrm{s}}$ estimate was $\sim 4$-fold higher for the $S D H B$ gene, indicating the very low frequency of the allelic variants. For comparison, $90 \%$ of the genes in a recent survey had $\theta_{\mathrm{s}}$ estimates higher than the $\pi$ estimates [28], indicating an abundance of rare alleles, which is thought to be a result of recent population expansion in humans. $\mathrm{F}_{\mathrm{ST}}$ statistics provided statistically significant evidence of population differentiation between the two racial groups for the $S D H A, S D H C$, and $S D H D$ genes, but not for the $S D H B$ gene (Table 2). This was attributable to the very low frequencies and the absence of $S D H B$ allelic variants in the African American and European American samples, respectively.

\section{Comparison of the human and chimpanzee SDHA genes for sequence diversity}

To test whether high nucleotide diversity also characterizes the chimpanzee SDHA gene, we used the human PCR primers to amplify and sequence 18 unrelated chimpan- zee samples. We obtained high-quality sequences for exons 3-6, 8, 12, 13, and 15, which together comprise a total genomic sequence size of 2832 bp (Table 1). We identified one silent exonic and seven intronic fixednucleotide differences between the human and chimpanzee SDHA genes (Additional file 2), corresponding to a substitution rate of $0.28 \%$. The nucleotide substitution rate in SDHA is lower than the average of 127 known genes $(0.75 \%)$ that were recently sequenced in human and chimpanzee [29]. The chimpanzee SDHA gene has 10 polymorphic variants, compared with 21 in the human gene in the same region, and showed $\sim 2$.9-fold lower nucleotide diversity $(\pi)$ than the human gene (Table 3 ). Furthermore, $\theta_{\mathrm{s}}$ and $\pi$ estimates of nucleotide diversities were similar in the chimpanzee, consistent with neutral expectations. These findings indicate that the mutation rate in SDHA is not inherently high and that the increased nucleotide diversity in the human gene must have occurred after the split of the two species from their common ancestor 5-6 million years ago.

\section{Tests of neutrality}

We employed three commonly used tests (the Tajima $D$ tests and the $\mathrm{Fu}$ and $\mathrm{Li} D^{*}$ and $F^{*}$ tests) to identify departures of the allelic distributions from neutral expectations. None of the PGL genes showed statistically significant departures from neutrality in samples from either racial group (Table 4). In contrast, the allelic distribution of the SDHA gene showed positive test values at statistically significant levels in both racial samples (Table 5). Notably, the neutrality statistics were supportive of balancing selection on SDHA despite the presence of six singleton variants in the African American samples and one singleton variant in the European American samples (Additional file 1). To obtain a clearer picture of the departure of SDHA allelic distributions from neutral expectations, we analyzed non-coding, coding, synonymous, and non-synonymous variants separately (Table 5). Nominally significant departures from neutrality were obtained in seven of the nine test statistics for the non-coding variants, although the SDHA coding region variation was also suggestive of an excess of variants in intermediate frequencies in the African American samples.

Table I: Summary of variants in SDH subunit genes

\begin{tabular}{llllll}
\hline Gene & $\begin{array}{l}\text { No. of coding base } \\
\text { pairs sequenced }\end{array}$ & $\begin{array}{l}\text { No. of synonymous } \\
\text { variants }\end{array}$ & $\begin{array}{l}\text { No. of non- } \\
\text { synonymous variants }\end{array}$ & $\begin{array}{l}\text { No. of non-coding } \\
\text { base pairs sequenced }\end{array}$ & $\begin{array}{l}\text { No. of non-coding } \\
\text { variants }\end{array}$ \\
\hline SDHA & 1995 & 12 & 3 & 3275 & 21 \\
SDHB & 843 & 1 & 2 & 879 & 0 \\
SDHC & 510 & 1 & 0 & 1303 & 7 \\
SDHD & 480 & 1 & 0 & 1556 & 4 \\
Chimpanzee SDHA & 1119 & 3 & 0 & 1713 & 7
\end{tabular}




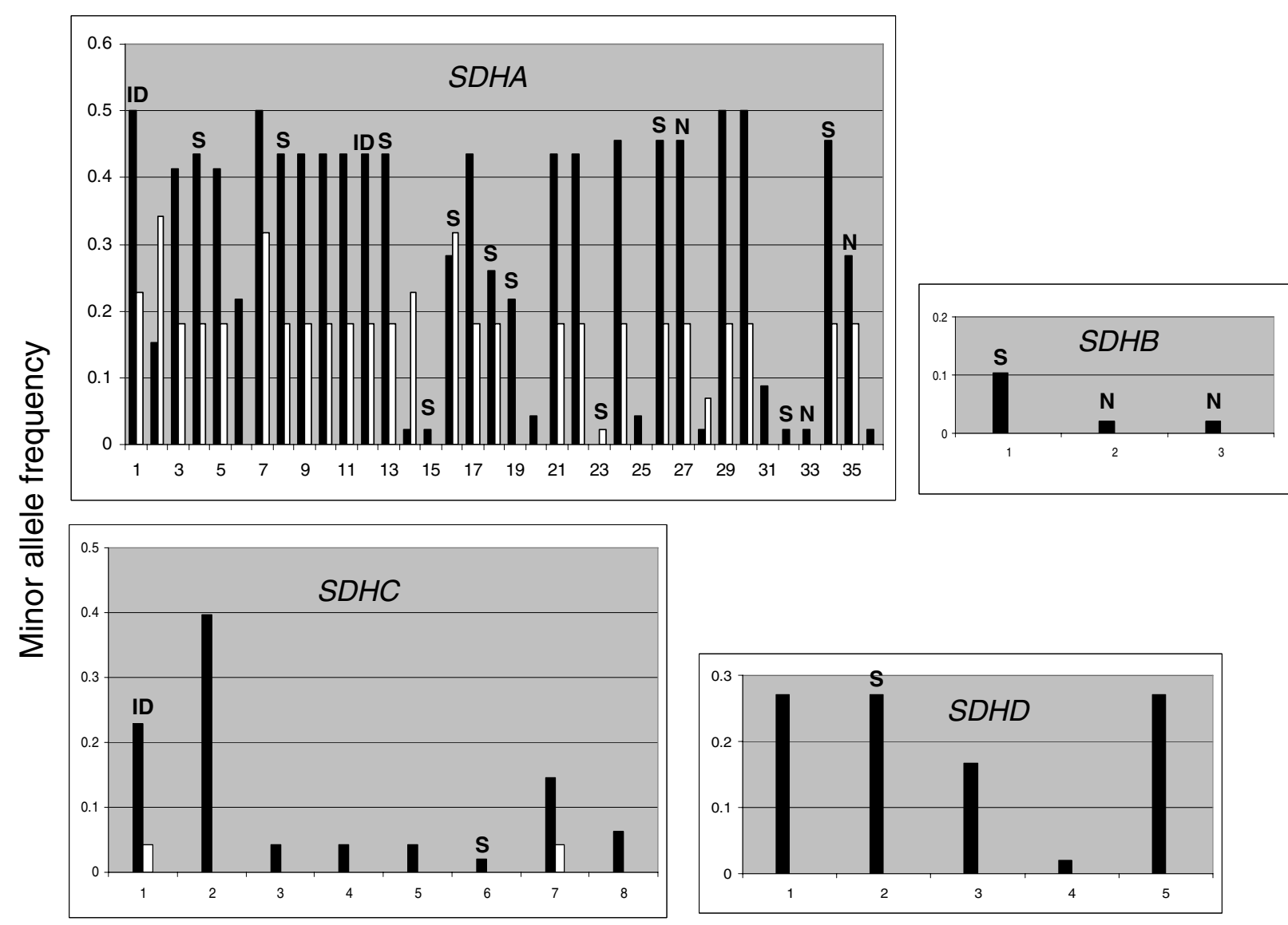

SNP No.

Figure I

Minor allele frequency of each SDH subunit variant (also see Additional Table I) is shown. Filled vertical bars refer to African American, unfilled vertical bars refer to European American samples. Synonymous and non-synonymous coding variants are marked by S and NS, respectively. ID refers to insertion/deletion polymorphisms.

To test whether the level of silent diversity in SDHA correlates with level of divergence between human and chimpanzee, as predicted by the neutral theory, we used the Hudson-Kreitman-Aguadé (HKA) test. Sequence data from four loci that were assumed to be evolving neutrally were used for comparison. These loci include non-coding regions on chromosome bands 1q24 [30], 22q11 [31], and Xq13.3 [32] and the promoter region of $\beta$-globin at 11 p15 [33]. Locus-by-locus comparison provided statistical significance in two of the four tests, suggesting increased diversity in SDHA relative to these two loci (Additional file 3). To further address whether the SDHA variation pattern is unusual when information from the comparison loci is jointly used, we used a recently developed maximum-likelihood-ratio test [34]. The likelihood of two models were compared; the first assumes that all five loci evolve neutrally, whereas the other assumes that $S D H A$ is subject to selection while the other four loci evolve neutrally. The model assuming selection on SDHA was statistically supported over the model of neutrality ( $p$ $=5.3 \times 10^{-3}$; Table 6 ). These results further support the hypothesis that increased nucleotide diversity in SDHA is maintained by balancing selection.

\section{Empirical assessment of neutrality in SDH subunit genes}

Because population history plays an important role in shaping the variation patterns in the genome, we sought to assess whether the nucleotide diversity of complex II genes were unusual compared with other genes across the genome. We used the summary statistics for Tajima's $D$ test and nucleotide diversity of 282 genes listed in the SeattleSNP database for comparison. When compared with the database genes, the statistics for nucleotide diversity and Tajima's $D$ were not outstanding for any of the complex II genes in the European American samples or for the SDHC and SDHD genes in the African American sam- 
Table 2: Sequence diversity in succinate dehydrogenase subunit genes

\begin{tabular}{|c|c|c|c|c|c|c|c|c|}
\hline Locus & Length (bp)* & Population & Sample size $(n) \dagger$ & $S \ddagger$ & $\begin{array}{l}\text { Gene diversity } \pm \\
\text { SD }\end{array}$ & $\begin{array}{l}\text { Nucleotide } \\
\text { diversity, \% } \\
(\theta \pm S D)\end{array}$ & $\begin{array}{l}\text { Nucleotide } \\
\text { diversity, \% } \\
(\pi \pm S D)\end{array}$ & $\mathrm{F}_{\mathrm{ST}}(\mathrm{P} \S)$ \\
\hline \multirow[t]{3}{*}{ SDHA } & 5270 & AA & 24 & 35 & $0.974 \pm 0.010$ & $0.141 \pm 0.045$ & $0.231 \pm 0.118$ & $0.093(0.012)$ \\
\hline & & EA & 24 & 27 & $0.903 \pm 0.030$ & $0.107 \pm 0.036$ & $0.147 \pm 0.077$ & \\
\hline & & All & 48 & 36 & $0.964 \pm 0.009$ & $0.126 \pm 0.037$ & $0.199 \pm 0.101$ & \\
\hline \multirow[t]{3}{*}{$S D H B$} & 1722 & AA & 24 & 3 & $0.231 \pm 0.078$ & $0.039 \pm 0.024$ & $0.016 \pm 0.019$ & $0.061(0.113)$ \\
\hline & & EA & 24 & 0 & 0 & 0 & 0 & \\
\hline & & All & 48 & 3 & $0.120 \pm 0.045$ & $0.034 \pm 0.021$ & $0.008 \pm 0.013$ & \\
\hline \multirow[t]{3}{*}{ SDHC } & 1813 & AA & 24 & 8 & $0.680 \pm 0.060$ & $0.086 \pm 0.040$ & $0.103 \pm 0.067$ & $0.176(\sim 0)$ \\
\hline & & EA & 24 & 2 & $0.082 \pm 0.053$ & $0.012 \pm 0.012$ & $0.013 \pm 0.017$ & \\
\hline & & All & 48 & 8 & $0.44 I \pm 0.06 I$ & $0.075 \pm 0.033$ & $0.065 \pm 0.046$ & \\
\hline \multirow[t]{3}{*}{$S D H D$} & 2036 & AA & 24 & 5 & $0.609 \pm 0.063$ & $0.055 \pm 0.028$ & $0.077 \pm 0.052$ & $0.234(\sim 0)$ \\
\hline & & EA & 24 & 0 & 0 & 0 & 0 & \\
\hline & & All & 48 & 5 & $0.361 \pm 0.059$ & $0.048 \pm 0.024$ & $0.044 \pm 0.034$ & \\
\hline
\end{tabular}

AA, African American samples; EA, European American samples; SD, standard deviation.

*Includes coding and non-coding sequences.

†Number of unrelated subjects.

$\ddagger$ Number of segregating sites.

$\S p$ value for population genetic structure between $A A$ and EA.

ples. However, the SDHA nucleotide diversity was higher than that of $279(p<0.015)$ of the genes and the Tajima $D$ statistic was higher than that of $281(p<0.0036)$ of the genes in the African American samples (Figure 2). In contrast, $S D H B$ had less sequence diversity than 280 of the SeattleSNP genes $(p<0.011)$ in the African American samples. A recent analysis of 151 loci in the SeattleSNP set has indicated that the $D$ statistic of the $A B O$ locus $(D=1.58)$ retains its significance in an African American population under several demographic scenarios [35]. Because the magnitude of $D$ in SDHA in our African American samples $(D=1.95)$ is higher than that in the $A B O$ locus (Figure 2), it is likely that the statistical support for balancing selection on SDHA would be retained by different population histories. In summary, the departure of SDHA allelic distribution from neutral expectations is empirically supported in the African American samples, consistent with a balancing selection mechanism.

\section{Haplotype structures of the SDH subunit genes}

Haplotypes, haplotype-block structures and the tagging SNPs for each block were inferred using the web-based
HAP software (see methods). As expected, the haplotypes were more variable in the African American than in the European American samples. The SDHA haplotype variation could be defined by 6 haplotype blocks and 13 tagging SNPs in the African American samples but only by 3 haplotype-blocks and 5 tagging SNPs in the European American samples (Additional file 1). In contrast, haplotype variation in the PGL genes could be defined by single-haplotype blocks. The most common haplotype accounted for $\sim 99 \%$ of the haplotypes of the PGL genes in the European American samples (Additional file 4). Similarly, the most common haplotype and its 1-nucleotide neighbors covered $~ 98 \%, 79 \%$ and $73 \%$ of the variation in the $S D H B, S D H C$, and $S D H D$ genes, respectively, in the African American samples.

The commonness of a single haplotype and its 1-nucleotide neighbors in the PGL genes was in stark contrast to the presence of two common but highly dissimilar haplotypes in SDHA in both racial groups. The two most common SDHA haplotypes, A1 and A2, accounted for 19\% (17/90) and $\sim 9 \%(8 / 90)$ of all haplotype diversity, respec-

Table 3: Sequence diversity in the human and chimpanzee SDHA genes

\begin{tabular}{clllll}
\hline Species & $\begin{array}{l}\text { Length } \\
(\mathrm{bp})\end{array}$ & $\begin{array}{l}\text { Sample size } \\
(n)\end{array}$ & $\mathrm{S}^{*}$ & $\begin{array}{l}\text { Nucleotide diversity, } \\
\%\left(\theta_{\mathrm{s}} \pm \mathrm{SD}^{\mathrm{c}}\right)\end{array}$ & $\begin{array}{l}\text { Nucleotide diversity, } \\
\%(\pi \pm S D)\end{array}$ \\
\hline Human & 2832 & & & & \\
AA & & 23 & 20 & $0.153 \pm 0.054$ & $0.279 \pm 0.147$ \\
EA & & 22 & 16 & $0.122 \pm 0.046$ & $0.168 \pm 0.093$ \\
All & & 45 & 21 & $0.139 \pm 0.045$ & $0.238 \pm 0.125$ \\
Chimpanzee & 2832 & 18 & 10 & $0.077 \pm 0.033$ & $0.082 \pm 0.051$
\end{tabular}

AA, African American samples; EA, European American samples; SD, standard deviation.

*Number of segregating sites. 
Table 4: Tests of Neutrality in PGL genes

\begin{tabular}{|c|c|c|c|c|c|c|c|c|c|}
\hline Locus & Length (bp)* & Population & $\begin{array}{l}\text { Sample size } \\
(n) \dagger\end{array}$ & $S \ddagger$ & $\% \theta_{\mathrm{s}}$ & $\% \pi$ & $\begin{array}{l}\text { Tajima } \\
\text { D test statistic }\end{array}$ & $\begin{array}{l}\text { Fu and } \mathrm{Li} \\
\mathrm{D}^{*} \text { test statistic }\end{array}$ & $\begin{array}{l}\mathrm{Fu} \text { and } \mathrm{Li} \\
\mathrm{F}^{*} \text { test statistic }\end{array}$ \\
\hline \multirow[t]{3}{*}{$S D H B$} & 1710 & AA & 24 & 3 & 0.04 & 0.016 & $-1.24(0.117)$ & $-1.70(0.152)$ & $-1.82(0.080)$ \\
\hline & 1710 & EA & 24 & 0 & 0 & 0 & -- & -- & -- \\
\hline & 1710 & All & 48 & 3 & 0.034 & 0.008 & $-1.37(0.07 \mid)$ & $-1.98(0.113)$ & $-2.10(0.044)$ \\
\hline \multirow[t]{3}{*}{$S D H C$} & 1806 & AA & 24 & 7 & 0.087 & 0.064 & $-0.724(0.266)$ & $0.472(0.186)$ & $0.107(0.463)$ \\
\hline & 1806 & EA & 24 & I & 0.012 & 0.005 & $-0.87(0.362)$ & $0.55(0.237)$ & $0.16(0.238)$ \\
\hline & 1806 & All & 48 & 7 & 0.075 & 0.039 & $-1.150(0.126)$ & $0.328(0.233)$ & $-0.20 I(0.4 I I)$ \\
\hline \multirow[t]{3}{*}{$S D H D$} & 2033 & AA & 24 & 5 & 0.055 & 0.076 & $0.887(0.166)$ & $0.144(0.282)$ & $0.438(0.329)$ \\
\hline & 2033 & EA & 24 & 0 & 0 & 0 & -- & -- & -- \\
\hline & 2033 & All & 48 & 5 & 0.048 & 0.044 & $-0.195(0.490)$ & $-0.017(0.334)$ & $-0.089(0.46 I)$ \\
\hline
\end{tabular}

AA, African American samples; EA, European American samples; SD, standard deviation. Significant $p$ values (within parentheses) are in bold.

*Includes coding and non-coding sequences.

†Number of unrelated subjects.

$\ddagger$ Number of segregating sites. DnaSp software excludes from the analyses the insertion/deletion variants, one of which is in SDHC.

tively, and differed from each other in 22 of the 36 variant positions (Figure 3). Haplotype A1 and A2 encode the missense Fp variants Y629-V657 and F629-657, by the SNPs 27 and 35, respectively, indicating an allelic association of the missense variants in these two amino-acid sites. Notably, the variant Fp amino acids Y629 and V657 were conserved in mammalian Fp sequences, including orangutan, macaque, mouse, dog, rat, and bovine. However, different amino acids were found in phylogenetically more distant species such as the zebrafish, which had Y629-I657 and Dirofilaria, an infectious nematode, which had E629-I657.

All of the remaining 34 SDHA haplotypes were highly similar to one of the two commonest haplotypes, and formed two distinct haplotype sets, referred to as haplogroup 1 and haplogroup 2. The haplotypes within each group differed from the most common haplotype of the group in up to seven variant positions, with a median number of three differences. The frequencies of haplogroups 1 and 2 were $~ 56 \%$ and $~ 44 \%$ in the African American samples and $\sim 82 \%$ and $\sim 18 \%$ in the European American samples, respectively. A median-joining network of all haplotypes clustered all but one haplotype within two distinct haplogroup clusters (Figure 4). The only haplotype (RR) that mapped outside of the two haplogroups clusters was probably a recombination product between haplogroup 1 and haplogroup 2 .

\section{Haplotype number test}

To test whether the number of predicted SDHA haplotypes in the African American samples is compatible with neutral evolution, we employed the Depaulis and Veuille haplotype number test [36]. In total, 35 variants in 46 African American sequences defined 27 different haplotypes (Figure 3). Using Depaulis and Veuille simulations under assumptions of neutrality showed that when there are 40 variants in 50 sequences, the upper limit of the $95 \%$ confidence interval for the expected number of different haplotypes is 24 . Thus, the number of SDHA haplotypes is statistically significantly higher than expected under neutrality, and is consistent with an ancient balanced polymorphism in the African American population.

\section{Estimating age of the SDHA haplogroups}

We estimated the age of the two haplogroups by comparing the sequence divergence between them with that between the human and chimpanzee genes, assuming a constant evolutionary rate of nucleotide substitutions. Haplogroups 1 and 2 have eight fixed nucleotide differences, at SNPs 8-12, 17, 21, and 22 (Figure 3), within 5255 bp, whereas human and chimpanzee genes have eight fixed nucleotide differences within 2832 bps. On the basis of these fixed nucleotide substitutions, we estimated haplogroups 1 and 2 to be as old as [(8/5255)/(8/2832)] times the divergence time of human and chimpanzees. Thus, SDHA balanced polymorphisms were estimated to be 2.69-3.23 million years old, assuming a divergence time of 5-6 million years for human and chimpanzees. This is probably a conservative estimate, as the fixed differences between the haplogroups erode in time by recombination and gene conversion.

\section{Discussion}

Our results establish a foundation to understand the selective and demographic forces that have shaped the variation patterns in SDH subunit genes, and have important functional implications. Our findings indicate that the variation pattern in SDHA is characterized by the presence of higher sequence diversity, two common and highly dissimilar haplogroups, and statistical and empirical support for the operation of a balancing selection mechanism. Our data also refute the previous suggestions 
Table 5: Tests of neutrality in SDHA

\begin{tabular}{|c|c|c|c|c|c|c|c|c|c|}
\hline Locus & Length (bp) & Population & Sample size $(n)^{*}$ & $\mathrm{~S} \dagger$ & $\% \theta_{\mathrm{s}}$ & $\% \pi$ & Tajima $D$ test statistic & $\begin{array}{l}\text { Fu and Li D* test } \\
\text { statistic }\end{array}$ & $\begin{array}{l}\text { Fu and Li } F^{*} \text { test } \\
\text { statistic }\end{array}$ \\
\hline \multirow[t]{3}{*}{ Coding } & 1995 & $\mathrm{AA}$ & 23 & 14 & 0.16 & 0.238 & $1.515(0.053)$ & $-0.352(0.428)$ & $0.320(0.367)$ \\
\hline & 1995 & EA & 22 & 10 & 0.115 & 0.147 & $0.797(0.181)$ & $0.805(0.098)$ & $0.945(0.154)$ \\
\hline & 1995 & All & 45 & 15 & 0.148 & 0.204 & $1.046(0.116)$ & $-1.065(0.210)$ & $-0.361(0.361)$ \\
\hline \multirow[t]{3}{*}{ Synonymous } & 1995 & $\mathrm{AA}$ & 23 & 11 & 0.125 & 0.191 & $\mathrm{I} .542(0.05 \mathrm{I})$ & $-0.249(0.491)$ & $0.399(0.344)$ \\
\hline & 1995 & EA & 22 & 8 & 0.092 & 0.116 & $0.726(0.192)$ & $0.612(0.416)$ & $0.761(0.215)$ \\
\hline & 1995 & All & 45 & 12 & 0.119 & 0.163 & $1.003(0.132)$ & $-0.988(0.235)$ & $-0.339(0.383)$ \\
\hline \multirow[t]{3}{*}{ Non-synonymous } & 1995 & $\mathrm{AA}$ & 23 & 3 & 0.034 & 0.047 & $0.810(0.197)$ & $-0.390(0.449)$ & $-0.036(0.495)$ \\
\hline & 1995 & EA & 22 & 2 & 0.023 & 0.031 & $0.597(0.23 \mathrm{I})$ & $0.76 \mathrm{I}(0.428)$ & $0.827(0.236)$ \\
\hline & 1995 & All & 45 & 3 & 0.03 & 0.041 & $0.687(0.215)$ & $-0.561(0.499)$ & $-0.199(0.453)$ \\
\hline \multirow{3}{*}{ Non-coding } & 3275 & AA & 23 & 19 & 0.133 & 0.217 & $2.046(0.015)$ & $0.908(0.084)$ & $1.534(0.036)$ \\
\hline & 3275 & EA & 22 & 15 & 0.106 & 0.145 & $1.176(0.092)$ & $1.565(0.038)$ & $1.696(0.020)$ \\
\hline & 3275 & All & 45 & 19 & 0.115 & 0.195 & $2.020(0.019)$ & $1.707(0.025)$ & $2.174(0.005)$ \\
\hline \multirow[t]{3}{*}{ Coding and non-coding } & 5255 & $\mathrm{AA}$ & 23 & 33 & 0.143 & 0.225 & $1.954(0.017)$ & $0.402(0.279)$ & $1.128(0.091)$ \\
\hline & 5255 & EA & 22 & 25 & 0.11 & 0.17 & $1.114(0.106)$ & $1.452(0.037)$ & $1.584(0.029)$ \\
\hline & 5255 & All & 45 & 34 & 0.128 & 0.198 & $1.721(0.036)$ & $0.521(0.228)$ & $1.183(0.079)$ \\
\hline
\end{tabular}

AA, African American samples; EA, European American samples; SD, standard deviation.

Significant $p$ values (within parentheses) are in bold.

*Number of unrelated subjects.

†Number of segregating sites. DnaSp software excludes from the analyses the insertion/deletion variants, two of which were detected in the noncoding SDHA sequence. Inclusion of the SDHA indels, by recoding them as SNPs, increased all SDHA test statistics and reduced the $p$ values (data not shown)

that the Y629F and V657I variants originate from two distinct genetic loci because these missense variants are encoded by a single, highly polymorphic SDHA gene.

The PGL genes had much lower nucleotide diversity, which was especially evident in $S D H B$, suggesting that the $S D H B$ gene product might be under functional constraints that preclude the accumulation of variants. If slightly deleterious variants in PGL genes increase the risk of paraganglioma tumor development, such variants would be eliminated before they reach high frequencies in the population. This potential mechanism might apply especially to $S D H B$ because its mutations are associated with malignancy and early-onset pheochromocytomas that could lead to severe hypertensive crises [37,38]. In contrast, because there is no evidence that heterozygous mutations in SDHA are associated with a pathologic phenotype, negative selection of deleterious SDHA alleles may operate only when they are in the homozygous state, which often leads to a lethal metabolic syndrome in childhood.
A major finding of our study is the unexpectedly high nucleotide diversity in the SDHA gene in the African American samples. It has been suggested that high local recombination rates may increase SNP density [39]. However, this mechanism is unlikely to contribute to SDHA variant density, because a recent high-resolution recombination map indicates a very low recombination rate at the tip of chromosome 5 short arm, where SDHA is located [40]. It is conceivable that the four SDHA pseudogenes, generated by complete or partial gene duplications, may increase the de novo mutation rate in the SDHA gene through illegitimate recombination or gene conversion during meiosis to increase variant density. However, lack of high nucleotide diversity in the chimpanzee SDHA gene does not suggest that the mutation rate in SDHA is inherently high, even though the chimpanzee genome also contains the duplicated SDHA pseudogenes. Rather, our findings suggest that the high nucleotide diversity of the human SDHA gene is a consequence of persistence of two distinct haplogroups for long periods during human evolution, leading to acquisition of a distinct set of polymorphisms by each haplogroup.

Table 6: Maximum-likelihood HKA analysis of silent polymorphisms in SDHA relative to four other neutrally evolving loci

\begin{tabular}{|c|c|c|c|c|c|c|c|c|c|}
\hline \multirow[t]{2}{*}{ Model } & \multirow[t]{2}{*}{ Description } & \multirow[t]{2}{*}{ Ln likelihood } & \multirow[t]{2}{*}{ Likelihood-ratio statistic (d.f.) } & \multirow[t]{2}{*}{$P$} & \multicolumn{5}{|l|}{$k^{*}$} \\
\hline & & & & & SDHA & $|q 2|$ & $\beta$-globin & $22 q 11$ & $\mathrm{Xq} / 3$ \\
\hline I & All neutral & -38.09 & -- & - & I & I & I & I & 1 \\
\hline 2 & Selection on SDHA & -34.2 & 7.777 (I) & $5.3 \times 10^{-3}$ & 4.7 & 1 & I & 1 & 1 \\
\hline
\end{tabular}

*Relative measure of diversity. 


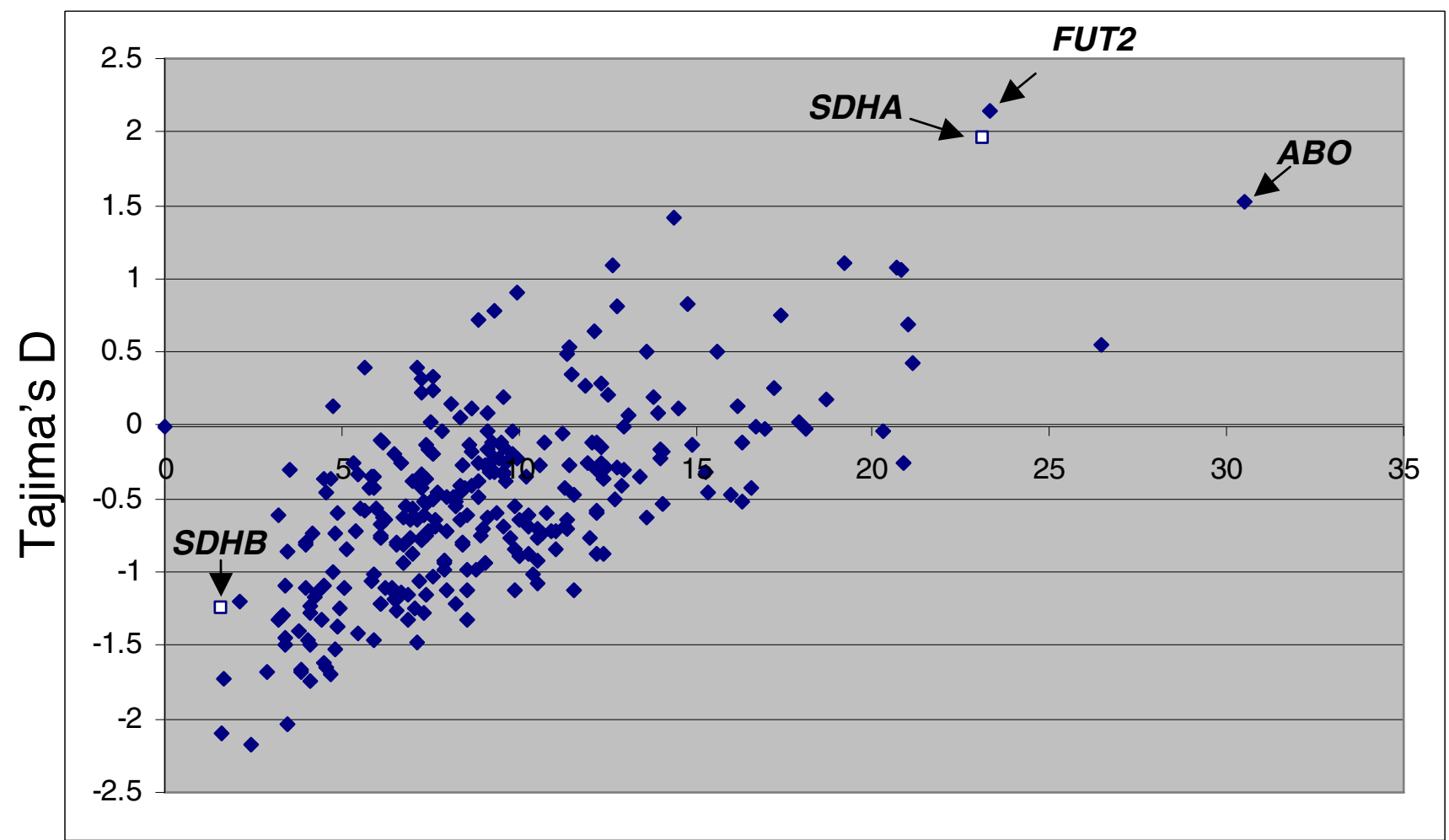

\section{Nucleotide diversity $\left(\mathrm{X} 10^{-4}\right)$}

\section{Figure 2}

Tajima's D statistic and nucleotide diversity in SDHA and SDHB genes (squares) relative to the 282 genes in SeattleSNP database are shown in the African American population. Positions of two highly polymorphic loci, $A B O$ and FUT2, the latter of which encodes the alpha(I,2)fucosyltransferase, are shown.

The most important finding of our study is the statistical and empirical support for a balancing selection mechanism on SDHA. A classic example of balancing selection is found at the major histocompatibility complex (MHC) loci [41], where high levels of polymorphisms in the functional MHC genes may confer a selective advantage to the heterozygotes by enabling them to process a wider range of pathogen antigens on $T$ cells. The variation in a few other human genes may also have been shaped by balancing selection. For example, the 5' cis-regulatory region of CCR5, encoding the principal coreceptor for HIV-1 [42], protocadherin alpha gene cluster promoters [43] and the bitter-taste receptor gene, PTC [44], have two major ancient haplotype groups and positive $D$ test statistics, similar to SDHA. However, in contrast to SDHA, these genes did not show significant Tajima $D$ statistics in the African or African American samples. In general, the average Tajima $D$ value is positive in the European American population and negative in the African American population. Positive Tajima $D$ statistics in European Americans are often interpreted to reflect population contraction that occurred during the migration of modern humans out of Africa, whereas negative Tajima $D$ statistics in African Americans may reflect admixture between African and European populations [35]. Thus, evidence of balancing selection on a gene, suggested by statistically significantly positive Tajima $D$ values, is more likely to be confounded by population history in European American samples than in African American samples.

It is conceivable that an environmental factor prevalent in Africa may have contributed to the increased frequency of certain SDHA variants that might have differential roles in the regulation of oxygen homeostasis by the SDH complex. A candidate environmental factor is the neurotoxin 3-NPA and its aliphatic nitrocompounds derivatives. In 


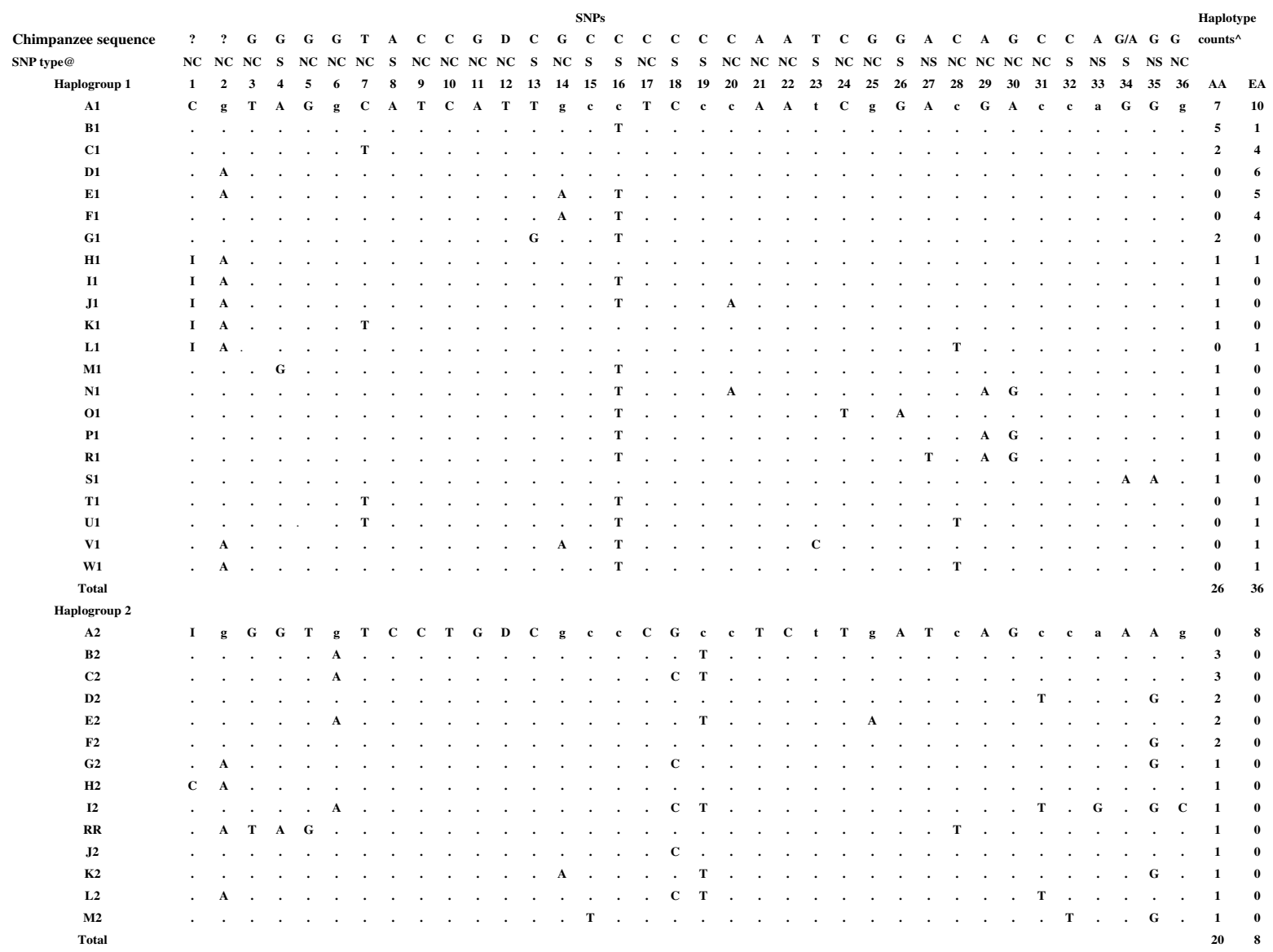

\section{Figure 3}

Haplotypes of SDHA. Periods denote the identical SNP variant when compared to the most common haplotype of each haplogroup. @NC = non-coding, $\mathrm{S}=$ synonymous coding, NS = non-synonymous coding. I = insertion allele, $\mathrm{D}=$ deletion allele. $\wedge \mathrm{AA}=$ African-American samples, EA = European-American samples.

addition to being a product of certain fungi such as Arthrinium species, 3-NPA and its derivatives are also found in several higher plants. The toxicity of these plants is well established, because their aliphatic nitrocompound contents have been linked to acute and chronic diseases in some domestic animals. Major livestock losses were attributed to plant nitrocompounds in the western United States, Canada and Mexico [45]. Thus, although human toxicity involving moldy sugarcane poisoning have to date been reported only in China, human exposure to 3-NPA and other nitrocompounds might be more common throughout the world than is indicated by the numbe of clinical cases $[18,46]$. 3-NPA exposure might be more prevalent in Africa partly because a hot and humid climate promotes the growth of fungi. If certain SDHA variants confer a selective advantage against 3-NPA poisoning by affecting gene expression levels, protein translation efficiency, and/or the binding affinity for 3-NPA, then such variants may provide a survival advantage for their carriers against 3-NPA poisoning. Alternatively, SDH may play a currently unrecognized role against infectious pathogens such as malaria, which are prevalent in Africa. Genetic studies of PGL suggest that inactivation of SDH by subunit mutations inappropriately activates hypoxiainducible pathways. If the SDHA variants that have increased in frequency during human evolution are hypomorphs or encode Fps that have slight functional deficits, these variants might promote the activation of hypoxiainducible pathways and help the immune cells to survive better under sustained hypoxic microenvironments of the infected tissues.

Finally, our findings do not support the previous explanations as to why SDHA mutations are not associated with PGL susceptibility because these explanations assume the presence of two SDHA genes in the human genome. 


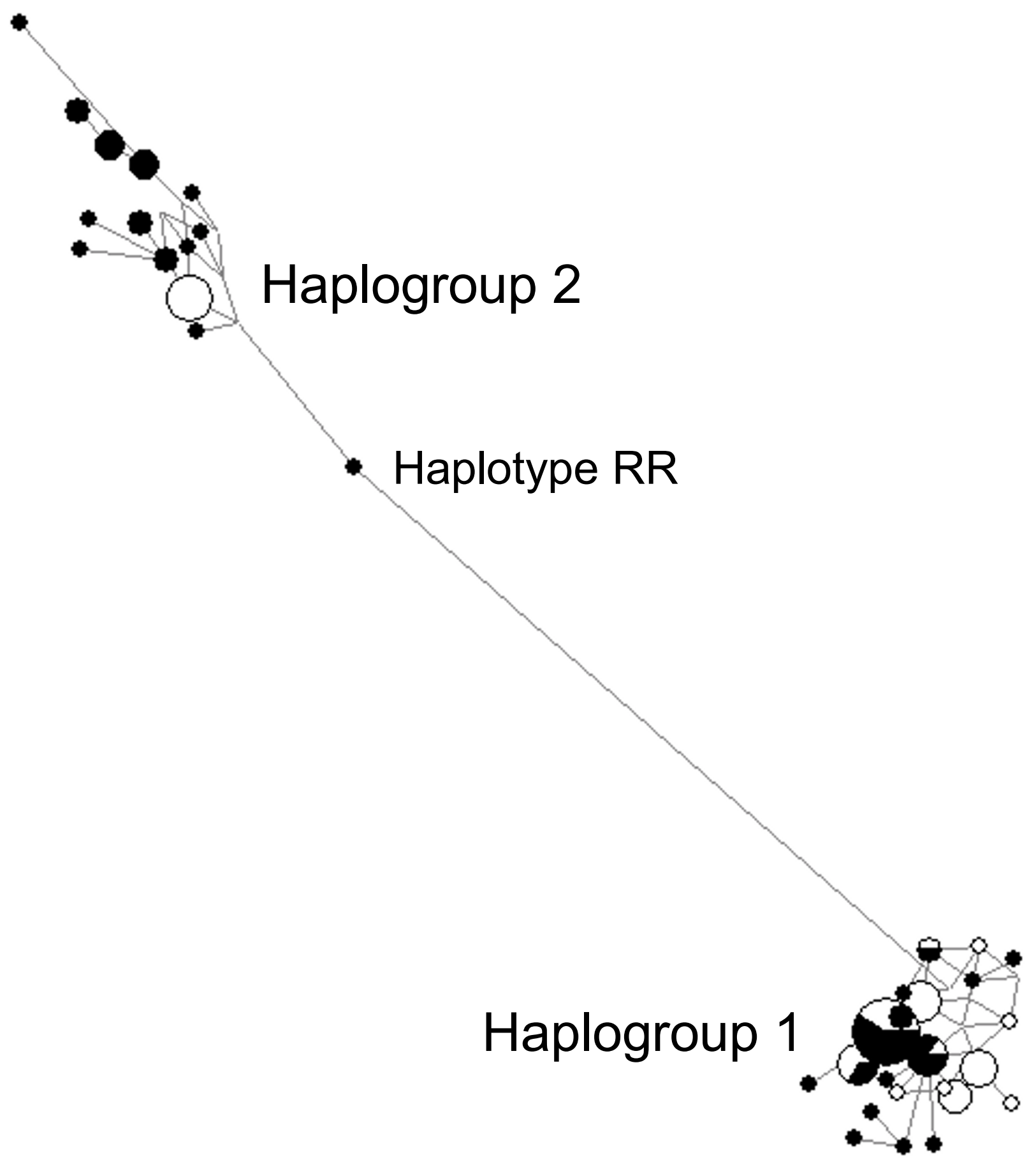

Figure 4

A median-joining network groups all SDHA haplotypes (Figure 3) on the basis of number of nucleotide differences. The haplotype RR is probably a recombinant between the two haplogroups. The pie chart for each haplotype depicts the proportional contribution of the African American (filled portion) and the European American (unfilled portion) samples. 
Instead, the contrasting patterns of sequence variation between SDHA and the PGL genes suggest the presence of two functionally distinct modules in SDH: one formed by the three closely-associated PGL gene products (PGL module), and the other a loosely-interacting, highly-variable SDHA protein product. This model provides an alternative explanation as to why SDHA mutations do not cause PGL and predicts the following two conditions:

(i) The relative concentration of SDHA protein product is much higher (>two-fold) than the PGL module in the paraganglionic tissues. Thus, even a 50\% reduction in SDHA protein levels, as a result of heterozygous mutations, would not compromise the SDH function in paraganglia to initiate tumor formation.

(ii) The physical interaction between the SDHA protein product and the PGL module is loose and kinetically fast during catalysis, thus a mutant SDHA protein product could not irreversibly trap a PGL module to initiate tumor formation.

\section{Conclusion}

Our findings demonstrate that the SDHA gene carries a strong signature of balancing selection in the African American population and that PGL and SDHA gene products are subject to distinct selective constraints. Collectively, these data provide new insights into SDH biology and may catalyze further research on the causes and the consequences of the unexpectedly high sequence diversity in the SDHA subunit gene.

\section{Methods \\ Samples}

DNA was isolated using standard protocols from samples from 24 unrelated African American and 24 unrelated European American women, which are part of an anonymized sample collection in the Department of Human Genetics at The University of Pittsburgh School of Public Health. The samples were collected under research protocols approved by the internal review board review committee. One African American and two European American samples that failed to amplify multiple SDHA exons on repeated attempts were removed from certain analyses, including minor allele frequency calculations, haplotype analysis, and neutrality statistics. We also sequenced the SDHA gene in 18 unrelated common chimpanzees (Pan troglodytes), which are part of the primate DNA collection in the Department of Human Genetics.

\section{PCR and sequencing}

PCR amplification for each exon was performed by using oligonucleotide primers that were designed from the flanking intronic or untranslated sequences of the exons. The primer sequences and the amplicon sizes for each
SDH subunit gene exon are provided in Additional file 5 . The PCR amplification was performed using Taq polymerase under standard conditions. The PCR amplification of SDHA is potentially confounded by the presence of multiple pseudogenes created by genomic duplications. These pseudogenes contain multiple mutations in their coding regions. BLAST analyses of human expressed sequences database in GenBank reveal no evidence for expression of the SDHA pseudogenes (data not shown). The PCR primers for specific amplification of the SDHA gene were designed so that the 3 ' ends of the primers were placed at nucleotides that showed divergence from the pseudogenes. The human genome March 2006 sequence assembly at UCSC database indicates that SDHA has two complete and one truncated gene duplications within $\sim 3$ $\mathrm{Mb}$ at chromosome band 3q29 and one truncated duplication $\sim 100 \mathrm{~kb}$ centromeric to the functional gene at chromosome band 5p15 [24]. The duplicated SDHA copies have $92.5-98.4 \%$ sequence identity with the functional gene within the exons and in the flanking introns. This high degree of sequence identity has erroneously led to the designation of some of the fixed nucleotide differences between the functional SDHA gene and its pseudogenes as real SNPs in the SDHA gene in the dbSNP database. In our experiments, we confirmed the specific amplification of each SDHA exon by analyzing the nucleotide positions of the amplicons where there are fixed differences between the functional and the duplicated gene copies (number of fixed nucleotide differences between SDHA and its duplicated pseudogenes are indicated in Additional file 5). In addition, we confirmed that all SDHA exonic variants, except the rare variants of SNPs 15 , 33 , and 36 , which were observed only once in our whole sample set (i.e. were singletons), are represented by multiple expressed sequence tags (ESTs) in the human EST database at NCBI as determined by BLAST analyses [47]. Taken together, these results confirm that our genomic primers have specifically amplified the exons of the functional SDHA gene while avoiding the duplicated pseudogenes.

\section{Computational analyses}

The sequenced segments of the genes, including the coding, non-coding and flanking intronic sequences, were conjoined in a single gene-sequence file. This file was then used to enter polymorphism data for each sample using Sequencher ${ }^{\mathrm{TM}}$ software (Gene Codes Corporation, Ann Arbor, MI, USA). The sequence files for each sample were used to generate input files for data analyses in population genetic software. Nucleotide diversity, population diversification analyses and departures from Hardy-Weinberg expectations were calculated using Arlequin software (version 2.001) [48]. Tests of neutrality were conducted using DnaSp software (version 4.10) [49]. The phylogenic relationship between the inferred haplotypes was estab- 
lished using Network software (version 4.1) [50]. All software programs were operated on a PC platform. Haplotype analyses and the prediction of tagging SNPs were performed using HAP, a free web-based haplotype analysis software.

\section{Sequence databases}

We used the BLAT function of UCSC genome browser to determine the genomic locations of and sequence similarities between SDHA genomic duplications [24]. The Ensembl genome browser was used to determine the intron-exon junction, transcription initiation sites, and start/stop codons of the SDH subunit genes [51]. Gene variation data in the SeattleSNP database (August 2006) derived from 24 African American individuals and 23 Europeans [52] were used to compare with our results.

\section{Population genetics}

\section{Nucleotide diversity}

Two measures of nucleotide diversity were derived using unphased genotypic data: $\pi$, which measures the mean number of differences per nucleotide between two randomly chosen sequences and $\theta_{\mathrm{s}^{\prime}}$ which measures the proportion of segregating sites under the assumption of an infinite site-neutral model. Both measures estimate the mutation rate, $\theta_{s}=4 \mathrm{~N}_{e} \mu$, where $\mathrm{N}_{\mathrm{e}}$ is the effective population size and $\mu$ is the neutral mutation rate per generation.

In a sample of $n$ chromosomes, $\pi=\Sigma_{i<j} \pi_{i, j} / n_{c^{\prime}}$ where $\pi_{i, j}$ is the number of nucleotide differences between $i$ th and $j$ th DNA sequences and $n_{c}=n(n-1) / 2$ and

$\theta_{\mathrm{s}}=\mathrm{S} / \mathrm{a}$, where $\mathrm{a}=\sum_{\mathrm{i}=1}^{\mathrm{n}-1} 1 / \mathrm{i}$

\section{Tests of neutrality}

$\theta_{\mathrm{s}}$ is strongly affected by the existence of deleterious alleles, because such alleles are usually present in low frequencies, but $\theta_{\mathrm{s}}$ is not affected by the frequency of mutants. Conversely, $\pi$ is not significantly affected by the presence of rare deleterious alleles because $\pi$ incorporates the frequency of mutants. If some of the variants in the sample have selective effects, then the estimates of $\theta_{\mathrm{s}}$ and $\pi$ will be different. Tajima [53] used the difference between these two estimates to detect selection among the sequences.

Tajima's $D$ statistic is calculated as $D=\left(\pi-\theta_{\mathrm{s}}\right) /[\operatorname{Var}(\pi$ $\theta_{\mathrm{s}} \mathrm{s}^{1 / 2}$

The value of $D$ is expected to be zero for selectively neutral variants in a constant population. A non-zero $D$ value is a sign of departure from the neutral model caused by a rel- ative excess (positive $D$ values) or deficiency (negative $D$ values) of substitutions of various frequencies [54].

Departures from the neutral model of the allelic distributions can also be tested by Fu and Li's $D^{*}$ and $F^{*}$ test statistics [55]. These tests compare the number of mutations between internal and external branches of a sequence genealogy with their expectations under selective neutrality. $D^{*}$ and $F^{*}$ tests compare the number of nucleotide variants observed only once in a sample with the total number of nucleotide variants and with the mean pairwise difference between the sequences, respectively. We assessed the significance of neutrality test statistics by comparing the observed test values to those obtained by 10000 coalescent simulations using sample size and number of segregating sites as variables and assuming a standard neutral model with no recombination. Coalescent simulations were performed by DnaSp software (version 4.10).

We used the HKA test for excesses of variation in SDHA gene. This test compares whether the level of intra-specific polymorphism parallels the level of nucleotide divergence between two species in a given locus relative to neutrally evolving loci. We used the direct HKA mode in the DNAsp software for locus-by-locus comparison. We also used a software testing maximum likelihood ratio of selection on SDHA in a multilocus framework as described previously [34]. Twice the difference of log likelihoods for two competing models is approximately $\chi^{2}$ distributed, with the degree of freedom (d.f.) equal to the number of selected loci. We seeded 100000 and 200000 cycles of the Markov chain to run two independent tests on a PC. Both chains provided similar results.

\section{Genetic structure of populations}

The genetic structure of populations was investigated by the analysis of molecular variance (AMOVA) approach, as implemented in Arlequin software [48]. This approach is based on the analyses of variance of gene frequencies. The proportion of total variation among populations is estimated by $\mathrm{F}_{\mathrm{ST}}$, Wright's fixation index.

\section{Haplotype analyses}

We used HAP, a software employing a highly accurate method for common haplotype prediction from genotype data [56] to calculate minor allele frequencies of all variants. The haplotype resolution employs a phasing method that uses imperfect phylogeny. This method partitions the SNPs into haplotype blocks, and for each block, it predicts the common haplotypes and each individual's haplotype. We used Network (version 4.1), a phylogenetic network analysis software, to generate an evolutionary tree network that links the predicted haplotypes on the basis of their similarity [50]. 


\section{Authors' contributions}

BEB and REF conceived and designed the study. BEB performed the statistical analyses and drafted the manuscript. REF and ECL revised the manuscript critically for important intellectual content. ECL performed the sequence analyses. BEB and REF obtained funding. All authors read and approved the final manuscript.

\section{Additional material}

\section{Additional file 1}

\section{Additional Table 1 - S DH subunit gene variants}

Click here for file

[http://www.biomedcentral.com/content/supplementary/1741-

7007-5-12-S1.doc]

\section{Additional file 2}

Additional Table 2 - Sequence variants and fixed differences in chimpanzee SDHA

Click here for file

[http://www.biomedcentral.com/content/supplementary/17417007-5-12-S2.doc]

\section{Additional file 3}

Additional Table 3 - Locus-by-locus HKA tests of SDHA versus neutrally evolving loci

Click here for file

[http://www.biomedcentral.com/content/supplementary/17417007-5-12-S3.doc]

\section{Additional file 4}

Additional Table 4 - Haplotype structures of SDHB, SDHC, and SDHD Click here for file

[http://www.biomedcentral.com/content/supplementary/17417007-5-12-S4.xls]

\section{Additional file 5}

Additional Table 5 - PCR oligonucleotide primers and the amplicon sizes for SDHA, SDHB, SDHC, and SDHD exons

Click here for file

[http://www.biomedcentral.com/content/supplementary/1741-

7007-5-12-S5.doc]

\section{Acknowledgements}

We thank Joan W. Willett-Brozick for technical help and three reviewers for helpful suggestions. This research is supported in part by a National Institute of Health grant CAII 2364 to BEB.

\section{References}

I. Scheffler IE: Molecular genetics of succinate:quinone oxidoreductase in eukaryotes. Prog Nucleic Acid Res Mol Biol 1998 60:267-315

2. Yankovskaya V, Horsefield R, Tornroth S, Luna-Chavez C, Miyoshi H, Leger C, Byrne B, Cecchini G, Iwata S: Architecture of succinate dehydrogenase and reactive oxygen species generation. Science 2003, 299:700-704.

3. Sun F, Huo X, Zhai Y, Wang A, Xu J, Su D, Bartlam M, Rao Z: Crystal structure of mitochondrial respiratory membrane protein complex II. Cell 2005, I 2 I: 1043-1057.
4. Baysal BE, Ferrell RE, Wilett-Brozick JE, Lawrence EC, Myssiorek D, Bosch A, van der Mey A, Taschner PEM, Rubinstein WS, Myers EN, Richard CW III, Cornelisse CJ, Devilee P, Devlin B: Mutations in SDHD, a mitochondrial complex II gene, in hereditary paraganglioma. Science 2000, 287:848-85I

5. Niemann S, Muller U: Mutations in SDHC cause autosomal dominant paraganglioma, type 3. Nat Genet 2000, 26:268-270.

6. Astuti D, Latif F, Dallol A, Dahia PL, Douglas F, George E, Skoldberg $F$, Husebye ES, Eng C, Maher ER: Gene mutations in the succinate dehydrogenase subunit sdhb cause susceptibility to familial pheochromocytoma and to familial paraganglioma. Am I Hum Genet 2001, 69:49-54.

7. Baysal BE, Willett-Brozick JE, Lawrence EC, Drovdlic CM, Savul SA, McLeod DR, Yee HA, Brackmann DE, Slattery WH III, Myers EN, Ferrell RE, Rubinstein WS: Prevalence of SDHB, SDHC, and SDHD germline mutations in clinic patients with head and neck paragangliomas. J Med Genet 2002, 39:।78-।83.

8. Baysal BE, Willett-Brozick JE, Filho PA, Lawrence EC, Myers EN, Ferrell RE: An Alu-mediated partial SDHC deletion causes familial and sporadic paraganglioma. J Med Genet 2004, 4I:703-709.

9. Bayley JP, Devilee P, Taschner PE: The SDH mutation database: an online resource for succinate dehydrogenase sequence variants involved in pheochromocytoma, paraganglioma and mitochondrial complex II deficiency. BMC Med Genet 2005, 6:39.

10. Schiavi F, Boedeker CC, Bausch B, Peczkowska M, Gomez CF, Strassburg T, Pawlu C, Buchta M, Salzmann M, Hoffmann MM, Berlis A, Brink I, Cybulla M, Muresan M, Walter MA, Forrer F, Valimaki M, Kawecki A, Szutkowski Z, Schipper J, Walz MK, Pigny P, Bauters C, Willet-Brozick JE, Baysal BE, Januszewicz A, Eng C, Opocher G, Neumann HP: Predictors and prevalence of paraganglioma syndrome associated with mutations of the SDHC gene. JAMA 2005, 294:2057-2063.

II. Dahia PL, Ross KN, Wright ME, Hayashida CY, Santagata S, Barontin M, Kung AL, Sanso G, Powers JF, Tischler AS, Hodin R, Heitritter S, Moore F, Dluhy R, Sosa JA, Ocal IT, Benn DE, Marsh DJ, Robinson BG, Schneider K, Garber J, Arum SM, Korbonits M, Grossman A, Pigny P, Toledo SP, Nose V, Li C, Stiles CD: A HIF I alpha regulatory loop links hypoxia and mitochondrial signals in pheochromocytomas. PLoS Genet 2005, I:72-80.

12. Selak MA, Armour SM, MacKenzie ED, Boulahbel H, Watson DG, Mansfield KD, Pan Y, Simon MC, Thompson CB, Gottlieb E: Succinate links TCA cycle dysfunction to oncogenesis by inhibiting HIF-alpha prolyl hydroxylase. Cancer Cell 2005, 7:77-85.

13. Piruat JI, Pintado CO, Ortega-Saenz P, Roche M, Lopez-Barneo J: The mitochondrial SDHD gene is required for early embryogenesis, and its partial deficiency results in persistent carotid body glomus cell activation with full responsiveness to hypoxia. Mol Cell Biol 2004, 24: 10933-10940.

14. Astrom K, Cohen JE, Willett-Brozick JE, Aston CE, Baysal BE: Altitude is a phenotypic modifier in hereditary paraganglioma type I: evidence for an oxygen-sensing defect. Hum Genet 2003, I I 3:228-237.

15. Alexi T, Hughes PE, Faull RL, Williams CE: 3-Nitropropionic acid's lethal triplet: cooperative pathways of neurodegeneration. Neuroreport 1998, 9:R57-R64.

16. Ming L: Moldy sugarcane poisoning - a case report with a brief review. J Toxicol Clin Toxicol 1995, 33:363-367.

17. Brouillet E, Jacquard C, Bizat N, Blum D: 3-Nitropropionic acid: a mitochondrial toxin to uncover physiopathological mechanisms underlying striatal degeneration in Huntington's disease. I Neurochem 2005, 95:1521-1540.

18. Ludolph AC, He F, Spencer PS, Hammerstad J, Sabri M: 3-Nitropropionic acid-exogenous animal neurotoxin and possible human striatal toxin. Can J Neurol Sci I99I, I 8:492-498.

19. Huang LS, Sun G, Cobessi D, Wang AC, Shen JT, Tung EY, Anderson VE, Berry EA: 3-nitropropionic acid is a suicide inhibitor of mitochondrial respiration that, upon oxidation by complex II, forms a covalent adduct with a catalytic base arginine in the active site of the enzyme. J Biol Chem 2006, 28 I:5965-5972.

20. Bourgeron T, Rustin P, Chretien D, Birch-Machin M, Bourgeois M, Viegas-Pequignot E, Munnich A, Rotig A: Mutation of a nuclear succinate dehydrogenase gene results in mitochondrial respiratory chain deficiency. Nat Genet 1995, I I: |44-| 49. 
21. Baysal BE, Rubinstein WS, Taschner PE: Phenotypic dichotomy in mitochondrial complex II genetic disorders. J Mol Med 200 I, 79:495-503.

22. Tomitsuka E, Goto Y, Taniwaki M, Kita K: Direct evidence for expression of type II flavoprotein subunit in human complex II (succinate-ubiquinone reductase). Biochem Biophys Res Com mun 2003, 3 I I:774-779.

23. Tomitsuka E, Hirawake $H$, Goto $Y$, Taniwaki M, Harada S, Kita K: Direct evidence for two distinct forms of the flavoprotein subunit of human mitochondrial complex II (succinate-ubiquinone reductase). J Biochem (Tokyo) 2003, I34:191-195.

24. UCSC genome browser 2006 [http://genome.ucsc.edu].

25. Briere JJ, Favier J, Benit P, El GV, Lorenzato A, Rabier D, Di Renzo MF, Gimenez-Roqueplo AP, Rustin P: Mitochondrial succinate is instrumental for HIF I alpha nuclear translocation in SDHAmutant fibroblasts under normoxic conditions. Hum $\mathrm{Mol}$ Genet 2005, I 4:3263-3269.

26. SDH mutation database 2006 [http://chromium.liacs.nl/ lovd sdh].

27. Cargill M, Altshuler D, Ireland J, Sklar P, Ardlie K, Patil N, Shaw N, Lane CR, Lim EP, Kalyanaraman N, Nemesh J, Ziaugra L, Friedland L, Rolfe A, Warrington J, Lipshutz R, Daley GQ, Lander ES: Characterization of single-nucleotide polymorphisms in coding regions of human genes. Nat Genet 1999, 22:231-238.

28. Stephens JC, Schneider JA, Tanguay DA, Choi J, Acharya T, Stanley SE, Jiang R, Messer CJ, Chew A, Han JH, Duan J, Carr JL, Lee MS, Koshy B, Kumar AM, Zhang G, Newell WR, Windemuth A, Xu C, Kalbfleisch TS, Shaner SL, Arnold K, Schulz V, Drysdale CM, Nandabalan K, Judson RS, Ruano G, Vovis GF: Haplotype variation and linkage disequilibrium in 313 human genes. Science 200I, 293:489-493.

29. Shi J, Xi H, Wang Y, Zhang C, Jiang Z, Zhang K, Shen Y, Jin L, Zhan K, Yuan W, Wang Y, Lin J, Hua Q, Wang F, Xu S, Ren S, Xu S, Zhao $G$, Chen Z, Jin L, Huang W: Divergence of the genes on human chromosome 2 I between human and other hominoids and variation of substitution rates among transcription units. Proc Natl Acad Sci USA 2003, 100:8331-8336.

30. Yu N, Zhao Z, Fu YX, Sambuughin N, Ramsay M, Jenkins T, Leskinen E, Patthy L, Jorde LB, Kuromori T, Li WH: Global patterns of human DNA sequence variation in a $\mathbf{1 0 - k b}$ region on chromosome I. Mol Biol Evol 2001, I 8:214-222.

31. Zhao Z, Jin L, Fu YX, Ramsay M, Jenkins T, Leskinen E, Pamilo P, Trexler M, Patthy L, Jorde LB, Ramos-Onsins S, Yu N, Li WH: Worldwide DNA sequence variation in a l0-kilobase noncoding region on human chromosome 22. Proc Natl Acad Sci USA 2000 , 97:11354-11358.

32. Kaessmann $H$, Heissig $F$, von Haeseler A, Paabo S: DNA sequence variation in a non-coding region of low recombination on the human X chromosome. Nat Genet 1999, 22:78-81.

33. Fullerton SM, Bond J, Schneider JA, Hamilton B, Harding RM, Boyce $\mathrm{AJ}$, Clegg JB: Polymorphism and divergence in the beta-globin replication origin initiation region. Mol Biol Evol 2000 , 17:179-188.

34. Wright SI, Charlesworth B: The HKA test revisited: a maximum-likelihood-ratio test of the standard neutral model. Genetics 2004, 1 68: 1071-1076.

35. Stajich JE, Hahn MW: Disentangling the effects of demography and selection in human history. Mol Biol Evol 2005, 22:63-73.

36. Depaulis F, Veuille M: Neutrality tests based on the distribution of haplotypes under an infinite-site model. Mol Biol Evol 1998 , I 5: 1788-1790

37. Young AL, Baysal BE, Deb A, Young WF Jr: Familial malignant catecholamine-secreting paraganglioma with prolonged survival associated with mutation in the succinate dehydrogenase B gene. I Clin Endocrinol Metab 2002, 87:4I0I-4I05.

38. Gimenez-Roqueplo AP, Favier J, Rustin P, Rieubland C, Crespin M, Nau V, Van Kien PK, Corvol P, Plouin PF, Jeunemaitre X: Mutations in the SDHB gene are associated with extra-adrenal and/or malignant phaeochromocytomas. Cancer Res 2003 63:5615-5621.

39. Lercher MJ, Hurst LD: Human SNP variability and mutation rate are higher in regions of high recombination. Trends Genet 2002, I 8:337-340.

40. Myers S, Bottolo L, Freeman C, McVean G, Donnelly P: A fine-scale map of recombination rates and hotspots across the human genome. Science 2005, 3 1 0:321-324.
41. Hughes AL, Nei M: Pattern of nucleotide substitution at major histocompatibility complex class I loci reveals overdominant selection. Nature 1988, 335:167-170.

42. Bamshad MI, Mummidi S, Gonzalez E, Ahuja SS, Dunn DM, Watkins WS, Wooding S, Stone AC, Jorde LB, Weiss RB, Ahuja SK: A strong signature of balancing selection in the $5^{\prime}$ cis-regulatory region of CCR5. Proc Natl Acad Sci USA 2002, 99: I 0539- 0544.

43. Noonan JP, Li J, Nguyen L, Caoile C, Dickson M, Grimwood J, Schmutz J, Feldman MW, Myers RM: Extensive linkage disequilibrium, a common 16.7-kilobase deletion, and evidence of balancing selection in the human protocadherin alpha cluster. Am J Hum Genet 2003, 72:621-635.

44. Wooding S, Kim UK, Bamshad MJ, Larsen J, Jorde LB, Drayna D: Natural selection and molecular evolution in PTC, a bitter-taste receptor gene. Am J Hum Genet 2004, 74:637-646.

45. Anderson RC, Majak W, Rassmussen MA, Callaway TR, Beier RC, Nisbet DJ, Allison MJ: Toxicity and metabolism of the conjugates of 3-nitropropanol and 3-nitropropionic acid in forages poisonous to livestock. J Agric Food Chem 2005, 53:2344-2350.

46. Peraica M, Domijan AM: Contamination of food with mycotoxins and human health. Arh Hig Rada Toksikol 200I, 52:23-35.

47. Basic Local Alignment Search Tool (BLAST) 2006 [http:// www.ncbi.nlm.nih.gov/BLAST/]

48. Schneider S, Roessli D, Excoffier L: Arlequin: A software for population genetics data analysis. Ver 2.000 Geneva 2000

49. Rozas J, Sanchez-DelBarrio JC, Messeguer X, Rozas R: DnaSP, DNA polymorphism analyses by the coalescent and other methods. Bioinformatics 2003, 19:2496-2497.

50. Bandelt HJ, Forster $\mathrm{P}$, Rohl A: Median-joining networks for inferring intraspecific phylogenies. Mol Biol Evol 1999, I 6:37-48.

5I. Ensembl genome browser 2006 [http://www.ensembl.org/ index.html].

52. SeattleSNP database 2006 [http://pga.gs.washington.edu/ summary stats.html].

53. Tajima F: Statistical method for testing the neutral mutation hypothesis by DNA polymorphism. Genetics 1989, I 23:585-595.

54. Bamshad M, Wooding SP: Signatures of natural selection in the human genome. Nat Rev Genet 2003, 4:99-III.

55. $\mathrm{Fu} \mathrm{YX}, \mathrm{Li} \mathrm{WH}$ : Statistical tests of neutrality of mutations. Genetics 1993, I 33:693-709.

56. Halperin E, Eskin E: Haplotype reconstruction from genotype data using Imperfect Phylogeny. Bioinformatics 2004 20:1842-1849.
Publish with Biomed Central and every scientist can read your work free of charge

"BioMed Central will be the most significant development for disseminating the results of biomedical research in our lifetime. "

Sir Paul Nurse, Cancer Research UK

Your research papers will be:

- available free of charge to the entire biomedical community

- peer reviewed and published immediately upon acceptance

- cited in PubMed and archived on PubMed Central

- yours - you keep the copyright
BioMedcentral 